\title{
Percepción de los familiares del paciente crítico acerca del apoyo psicosocial que brinda la enfermera en la Unidad de Cuidados Intensivos del Hospital Nacional Guillermo Almenara Irigoyen
}

\author{
Perceptions of relatives of critically ill patients for psychosocial support provided by the nurse in the \\ Intensive Care Unit of the Guillermo Almenara Irigoyen Nacional Hospital
}

Manuel Reyes Tiburcioํㅜ, Karina Margoth Colonia Jáuregui ${ }^{2}$ Ruth Evelin Reyes Salas ${ }^{3}$

\begin{abstract}
RESUMEN
Objetivo: Determinar la percepción de los familiares del paciente crítico sobre el apoyo psicosocial que brinda la enfermera en la Unidad de Cuidados Intensivos del Hospital Nacional Guillermo Almenara Irigoyen. Material y métodos: El estudio fue de tipo descriptivo y de corte transversal. La muestra estuvo conformada por 50 familiares de los pacientes hospitalizados en la UCI, determinada mediante un muestreo probabilístico aleatorio simple. La recolección de datos se realizó mediante un cuestionario con 19 ítems, elaborado por los autores. Resultados: El 64\% de los familiares percibe favorablemente el apoyo psicosocial que brinda la enfermera en la UCI y el $36 \%$ lo percibe en forma desfavorable. En relación al aspecto emocional, se encontró que en las dimensiones de comprensión y tolerancia en la visita, y escucha al familiar, el 96\% tiene una percepción de favorable a muy favorable; asimismo en la dimensión de expresiones de afecto y apoyo, el $92 \%$ tiene también una percepción de favorable a muy favorable. En el aspecto instrumental, se evidencia que los familiares tienen una percepción de favorable a muy favorable en las dimensiones de información sobre necesidades específicas y complementarias, información en forma clara y sencilla, e información sobre los medios diagnósticos con un $100 \%, 94 \%$ y $52 \%$ respectivamente. En relación a la solución de problemas específicos, se reporta que el $70 \%$ tiene una percepción de favorable a muy favorable en la dimensión de participación de los familiares en el cuidado del paciente. Por el contrario, el 60\% tiene una percepción indiferente en la dimensión de información sobre requisitos para reposición de sangre, y el 50\% tiene una percepción de desfavorable a muy desfavorable en la dimensión de flexibilidad en la visita a los familiares. Conclusión: Se concluye que la percepción que tienen los familiares del paciente crítico sobre el apoyo psicosocial que brinda la enfermera en la UCI es favorable.
\end{abstract}

Palabras clave: Percepción, apoyo psicosocial, familiares, paciente crítico.

\section{SUMMARY}

Objective: To determine the perceptions of relatives of critically ill patients on the psychosocial support provided by the nurse in the Intensive Care Unit of the Hospital Nacional Guillermo Almenara Irigoyen. Material and methods: Study was descriptive and cross-sectional. The sample consisted of 50 family members of patients hospitalized in the ICU, determined by random probability sampling. Data collection was performed using a questionnaire with 19 items, elavorado by the authors. Results: $64 \%$ of household favorably perceived psychosocial support provided by the nurse in the ICU and 36\% perceived unfavorably. Regarding the emotional aspect, it was found that the dimensions of understanding and tolerance in the visit, and listen to the family, $96 \%$ have a favorable perception of very favorable, likewise in the size of expressions of affection and support, $92 \%$ also have a favorable perception of very favorable. In the instrumental aspect, evidence that family members have a favorable perception of very favorable dimensions and additional information on specific needs, information clearly and simply, and information on the diagnostic with $100 \%, 94 \%$ and $52 \%$ respectively. Regarding the solution of specific problems, it is reported that $70 \%$ have a favorable perception of very favorable in the dimension of family involvement in the care of the patient. By contrast, $60 \%$ have an indifferent perception in the dimension

\footnotetext{
${ }^{1}$ Enfermero asistencial del servicio de Emergencia de la Clínica Maisón de Santé. Enfermero asistencial en el servicio de UCIN del Hospital Alberto Sabogal de EsSalud, Lima, Perú.

${ }^{2}$ Enfermera asistencial del área de Recuperación Posanestésica de la Clínica Oncológica de Miraflores. Enfermera asistencial en el área ambulatoria del Hospital Militar Central, Lima, Perú.

${ }^{3}$ Enfermero asistencial en la Clínica San Gabriel, Lima, Perú.
} 
of information about requirements for blood replacement, and 50\% have an unfavorable perception of a very unfavorable in the dimension of flexibility in visiting relatives. Conclusion: It is concluded that the perception relatives of critically ill patients on the psychosocial support provided by the nurse in the ICU is favorable.

Keywords: Perception, psychosocial support, family, critical patient.

\section{INTRODUCCIÓN}

La familia es la primera célula social en el proceso de salud/enfermedad de sus miembros, todo proceso de enfermedad que afecte a uno de sus integrantes, altera, por lo general la estabilidad que disfrutan, obligándoles a ajustarse a la nueva situación. (Machado, 2007).

La orientación de los servicios sanitarios debe incorporar la atención a la familia como un elemento clave en el cuidado del enfermo; el ingreso de un enfermo en una Unidad de Cuidados Intensivos, supone una barrera física y psicológica en la vida de interrelación familia-paciente. El aislamiento del enfermo-familia tiene repercusiones para ambos y de forma indirecta influye también negativamente en el sistema y sobre la familia se produce un aumento de los niveles de ansiedad. (Triviño, 2011).

Esto es debido a que los problemas individuales de salud no se centran en el individuo en sí, sino que trascienden y afectan a la familia como grupo. El interés del estudio consiste en clarificar hasta qué punto se percibe el apoyo psicosocial en la práctica profesional de enfermería; asimismo integrar al individuo y a su familia al proceso de cuidados se hace fundamental y se justifica en base a varios postulados. (Lastra, 2004).

Por otra parte, la Asociación Americana de Psiquiatría, califica la hospitalización en una unidad de cuidados intensivos como un estrés traumático, ya que somete a los miembros de una familia a una difícil situación que implica cambios de roles, desplazamiento del hogar y miedo a la muerte; la familia se ve obligada a compaginar sus sentimientos de incertidumbre con la comprensión de explicaciones, la toma de decisiones difíciles, y, en ocasiones, con la aceptación de lo inevitable. Estos conflictos afectivos y emocionales pueden repercutir no solo en la salud psicológica de la familia sino, además, en la recuperación del paciente. (García, 2007).

Mendoza y Warren (1998), realizaron un estudio exploratorio en 52 miembros de la familia entre $18 \mathrm{y}$ 80 años dentro de las 18 y 24 horas de internamiento de su paciente en la Unidad de Cuidados Intensivos, donde encontraron que la seguridad, proximidad y la información son las necesidades de más importancia para la familia.
La familia es un sistema abierto y funciona como unidad. La aplicación de la Teoría General de los Sistemas a la familia muestra la necesidad de centrar en ella la atención. En efecto, según dicha teoría, un sistema es una complejidad organizada cuyo comportamiento está determinado por la interacción entre varios componentes. (Torres, 2004).

La familia experimenta sentimientos y reacciones emocionales de diferente intensidad tales como miedo, ansiedad, culpa, enojo, angustia, entre otros; es decir afronta una crisis situacional de manera súbita ante la enfermedad de su pariente enfermo requiriendo para ello de una intervención oportuna de la enfermera, Por ello, el desarrollo científico y tecnológico de enfermería, no puede menoscabar el profundo sentido y espíritu humanitario del cuidado de enfermería que brinda a las personas y a la sociedad. Ya que con frecuencia se observa que el familiar expresa: "cuando le preguntamos a la enfermera ella está siempre atenta y nos responde las dudas", "se interesa por informarnos al momento de la visita", "la enfermera me apoya con la visita porque me deja pasar aun en horarios no indicados".

La familia percibe un aumento en la calidad del cuidado al proporcionarles una mayor información. En palabras de Lastra, 2004: "Las enfermeras debemos hacer un esfuerzo para ir integrando a los familiares en los cuidados básicos del paciente".

El propósito estuvo orientado a contar con información real y objetiva sobre la percepción del familiar respecto a la intervención de enfermería durante el apoyo psicosocial que permita que el departamento de Enfermería formule y/o elabore estrategias de capacitación dirigida al personal de enfermería tendiente a reforzar las áreas críticas y reorientar su labor como agente terapéutico en el abordaje ante la crisis psicosocial que experimenta la familia favoreciendo así la relación enfermera-familiar.

El objetivo del estudio es determinar la percepción de los familiares acerca del apoyo psicosocial que brindan las enfermeras de la Unidad de Cuidados Intensivos del Hospital Nacional Guillermo Almenara Irigoyen.

\section{MATERIAL Y MÉTODOS}

Es un estudio de tipo descriptivo, transversal, con un enfoque cuantitativo. La población estuvo 
conformada por 53 pacientes internados en el mes de marzo - mayo en la Unidad de Cuidados Intensivos del Hospital Nacional Guillermo Almenara Irigoyen, del cual se extrajo la muestra conformada por 50 familiares que asistieron a las visitas de los pacientes hospitalizados en la unidad del mencionado hospital, para esta selección se utilizó el muestreo probabilístico aleatorio simple.

La recolección de datos se realizó mediante la aplicación de un cuestionario estructurado individual, en forma autoadministrada por los familiares, elaborado por los investigadores y que fue validado mediante juicio de cinco expertos.

Para su aplicación se realizó una prueba piloto a 15 familiares que asistieron a la visita de un pariente hospitalizado en la unidad de cuidados intensivos del mencionado hospital obteniendo una confiabilidad de $\alpha=0.95$ según el coeficiente "Alfa de Cronbach".
El cuestionario de 19 afirmaciones, todas en positivo, a las respuestas de los familiares se les asignó un puntaje de 5 a 1 en la Escala de Lickert, del cual pudo obtenerse puntajes parciales y totales.

Para la clasificación de la percepción, se asignó a cada ítem con percepción favorable 5 puntos y al ítem con percepción muy desfavorable, 0 puntos, totalizando 95 puntos en el caso ideal.

Los datos obtenidos fueron procesados en el sistema SPSS versión 19.0, realizándose la elaboración de tablas de frecuencia y gráficos para la presentación de los resultados.

Se demostró asociación estadísticamente significativa mediante la prueba del chi cuadrado $(p<$ de $0,05)$.

\section{RESULTADOS}

Tabla 1

Características de los familiares de pacientes de la Unidad de Cuidados Intensivos del Hospital Guillermo Almenara Irigoyen, marzo - mayo, 2012.

\begin{tabular}{|c|c|c|c|}
\hline \multicolumn{2}{|c|}{ Características de los familiares } & \multirow{2}{*}{$\frac{n}{6}$} & \multirow{2}{*}{$\begin{array}{l}\% \\
12,0 \%\end{array}$} \\
\hline \multirow{4}{*}{ Edad } & 18 - 28 años & & \\
\hline & $29-38$ años & 9 & $18,0 \%$ \\
\hline & $39-48$ años & 14 & $28,0 \%$ \\
\hline & 49 - 58 años & 12 & $24,0 \%$ \\
\hline \multirow{3}{*}{ Sexo } & 59 a más años & 9 & $18,0 \%$ \\
\hline & Hombre & 21 & $42,0 \%$ \\
\hline & Mujer & 29 & $58,0 \%$ \\
\hline \multirow{6}{*}{ Parentesco } & Cónyuge & 3 & $6,0 \%$ \\
\hline & Hijo (a) & 11 & $22,0 \%$ \\
\hline & Padre - madre & 11 & $22,0 \%$ \\
\hline & Hermano (a) & 15 & $30,0 \%$ \\
\hline & Amigo (a) & 3 & $6,0 \%$ \\
\hline & Otros & 7 & $14,0 \%$ \\
\hline \multirow{4}{*}{ Grado de instrucción } & Analfabeto & 0 &, $0 \%$ \\
\hline & Primaria & 4 & $8,0 \%$ \\
\hline & Secundaria & 26 & $52,0 \%$ \\
\hline & Técnico & 5 & $10,0 \%$ \\
\hline
\end{tabular}

En la tabla 1 se observa el $28 \%$ de una muestra que correspondió al grupo etario de 39 a 48 años; el 58\% fueron mujeres y el $42 \%$ varones; el $30 \%$ corresponde al grado de parentesco de hermana, el $52 \%$ cuenta con el grado de instrucción de nivel secundario. 


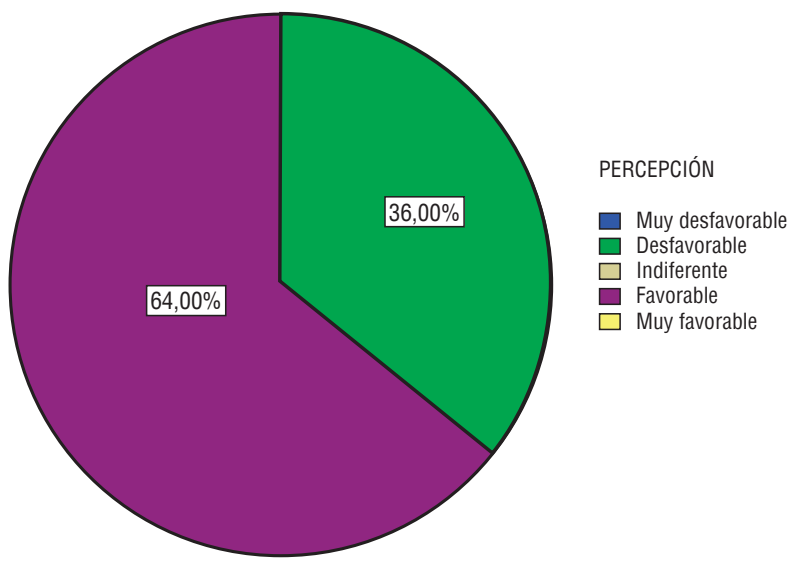

\section{Figura 1}

Familiares de pacientes de la Unidad de Cuidados Intensivos según percepción global del apoyo psicosocial brindado por las enfermeras en el Hospital Guillermo Almenara Irigoyen.

En la figura 1 se observa que la percepción de los familiares en relación al apoyo psicosocial brindado por la enfermera en la Unidad de Cuidados Intensivos, es favorable en un $64 \%$ y desfavorable en un $36 \%$.

\section{Tabla 2}

Familiares de pacientes de la UCI según la percepción del apoyo emocional brindado por las enfermeras en el aspecto emocional.

\begin{tabular}{clcc}
\hline Apoyo Emocional & & N & $\%$ \\
\hline & M. desfavorable & - & $0 \%$ \\
ESCUCHA A SU & Desfavorable & 1 & $2,0 \%$ \\
FAMILIAR & Indiferente & 1 & $2,0 \%$ \\
& Favorable & 30 & $60,0 \%$ \\
& M. favorable & 18 & $36,0 \%$ \\
\cline { 2 - 4 } MANIFIESTA & M. desfavorable & 0 & $0 \%$ \\
EXPRESIONES DE & Desfavorable & 0 & $0 \%$ \\
APOYO & Indiferente & 4 & $8,0 \%$ \\
& Favorable & 32 & $64,0 \%$ \\
PERCIBE & M. favorable & 14 & $28,0 \%$ \\
\cline { 2 - 4 } & M. desfavorable & 0 & $0 \%$ \\
TOLERANCIA & Desfavorable & 2 & $4,0 \%$ \\
& Indiferente & 0 & $0 \%$ \\
& Favorable & 36 & $72,0 \%$ \\
& M. favorable & 12 & $24,0 \%$ \\
\hline & Total & $100.0 \%$
\end{tabular}

En la tabla 2 se encontró que en las dimensiones de comprensión y tolerancia en la visita, y escucha al familiar, el 96\% tiene una percepción de favorable a muy favorable; asimismo en la dimensión de expresiones de afecto y apoyo, el 92\% tiene también una percepción de favorable a muy favorable. 
Tabla 3

Familiares de pacientes de la UCI según la percepción del apoyo psicosocial brindado por las enfermeras en el aspecto instrumental.

\begin{tabular}{clcc}
\hline Aspecto Instrumental & & $\mathbf{N}$ & $\%$ \\
\hline \multirow{2}{*}{ INFORMACIÓN SOBRE LOS } & M. desfavorable & 0 &, $0 \%$ \\
MEDIOS DIAGNÓSTICOS & Desfavorable & 2 & $4,0 \%$ \\
& Indiferente & 22 & $44,0 \%$ \\
& Favorable & 13 & $26,0 \%$ \\
& M. favorable & 13 & $26,0 \%$ \\
\cline { 2 - 4 } INFORMACIÓN SOBRE & M. desfavorable & 0 &, $0 \%$ \\
NECESIDADES ESPECÍFICAS Y & Desfavorable & 0 &, $0 \%$ \\
COMPLEMENTARIAS & Indiferente & 0 &, $0 \%$ \\
& Favorable & 22 & $44,0 \%$ \\
INFORMACIÓN EN FORMA & M. favorable & 28 & $56,0 \%$ \\
\cline { 2 - 4 } CLARA, OPORTUNA Y SENCILLA & M. desfavorable & 0 &, $0 \%$ \\
& Desfavorable & 2 & $4,0 \%$ \\
& Indiferente & 1 & $2,0 \%$ \\
& Favorable & 29 & $58,0 \%$ \\
\cline { 2 - 4 } & M. favorable & 18 & $36,0 \%$ \\
\cline { 2 - 4 } & Total & 50 & $100.0 \%$
\end{tabular}

En la tabla 3 se evidencia que los familiares tienen una percepción de favorable a muy favorable en las dimensiones de información sobre necesidades específicas y complementarias, información en forma clara y sencilla, e información sobre los medios diagnósticos con un $100 \%, 94 \%$ y $52 \%$ respectivamente.

Tabla 4

Familiares de pacientes de la UCI según la percepción del apoyo psicosocial brindado por las enfermeras en la solución de problemas.

\begin{tabular}{clcc}
\hline Solución de Problemas & & $\mathbf{N}$ & $\%$ \\
\hline \multirow{3}{*}{ FLEXIBILIDAD EN LA VISITA DE } & M. desfavorable & 12 & $24,0 \%$ \\
LOS FAMILIARES & Desfavorable & 13 & $26,0 \%$ \\
& Indiferente & 5 & $10,0 \%$ \\
& Favorable & 12 & $24,0 \%$ \\
& M. favorable & 8 & $16,0 \%$ \\
\cline { 2 - 4 } PARTICIPACIÓN DE LOS & M. desfavorable & 1 & $2,0 \%$ \\
FAMILIARES EN EL CUIDADO DEL & Desfavorable & 8 & $16,0 \%$ \\
PACIENTE & Indiferente & 6 & $12,0 \%$ \\
& Favorable & 25 & $50,0 \%$ \\
PNFORMACIÓN SOBRE REQUISITOS REPOSICIÓN DE SANGRE & M. favorable & 10 & $20,0 \%$ \\
\cline { 2 - 4 } & M. desfavorable & 0 &, $0 \%$ \\
& Indiferente & 5 & $10,0 \%$ \\
& Favorable & 30 & $60,0 \%$ \\
& Muy favorable & 7 & $14,0 \%$ \\
& Total & 8 & $16,0 \%$ \\
\hline
\end{tabular}


En la tabla 4 se reporta que el $70 \%$ tiene una percepción de favorable a muy favorable en la dimensión de participación de los familiares en el cuidado del paciente. Por el contrario, el $60 \%$ tiene una percepción indiferente en la dimensión de información sobre requisitos para reposición de sangre, y el 50\% tiene una percepción de desfavorable a muy desfavorable en la dimensión de flexibilidad en la visita a los familiares.

\section{DISCUSIÓN}

La percepción global de los familiares encuestados es favorable, con el $64 \%$, esto en lo que se refiere al apoyo psicosocial que brinda el enfermero de la UCI se explica porque estableció contacto con la familia, al mismo tiempo que le trasmite confianza, poniéndose en el lugar de la familia, comprendiendo que hizo un gran esfuerzo por visitar a su familiar. Según Bacigalupo (2000), la familia se ve obligada a compaginar sus sentimientos de incertidumbre con la comprensión de explicaciones, la toma de decisiones difíciles y en ocasiones, con la aceptación de lo inevitable.

En sentido divergente a los hallazgos del presente estudio, Franco (2008), en un estudio sobre la percepción familiar del paciente crítico en el Hospital Edgardo Rebagliati Martins en el 2003, observó que la percepción del familiar del paciente crítico respecto a la intervención de enfermería durante la crisis situacional tuvo una tendencia hacia lo desfavorable con un $38.8 \%$ e indiferente $27.7 \%$ en familiares entrevistados en adultos jóvenes y adultos maduros.

En las dimensiones del apoyo psicosocial, los familiares percibieron favorable y muy favorablemente el papel que realiza la enfermera de la unidad de cuidados intensivos, en lo que se refiere al aspecto emocional en las dimensiones de comprensión y tolerancia en la visita, escucha familiar, expresión de afecto y apoyo que brinda la enfermera. Estas conductas demuestran que la enfermera apoya a los familiares emocionalmente en el momento de la visita; resultados que coinciden con Franco (2003), quien resalta que el apoyo emocional está referido a facilitar la expresión de emociones del familiar, a través de la confianza, el contacto físico, dar ánimo, dar la mano al saludar, un abrazo o una suave palmada en el hombro, mostrar preocupación e interés por su sentir, actitud amigable en la que los familiares sientan la confianza y puedan expresar sus sentimientos a fin de calmar su ansiedad.

Los familiares percibieron favorablemente el apoyo psicosocial en el aspecto instrumental que brinda la enfermera, dado que la enfermera previamente les informa sobre las necesidades específicas como llevar útiles de aseo, pañales y otros complementos como aditamentos o dispositivos que favorezcan el cuidado y recuperación de su paciente.

El 58\% de la muestra percibió de forma clara y sencilla la información brindada por la enfermera intensivista; el 50\% de los familiares percibe que se valoran las necesidades espirituales de sus pacientes, se les permite el ingreso de rosarios o imágenes del santo de su devoción; un 44\% de la muestra percibe con duda si se le comunica o no algún procedimiento diagnóstico que requiera su familiar; estos hallazgos coinciden con el estudio realizado por Santana (2005), donde el $100 \%$ de los familiares afirmó haber comprendido la información que recibieron al ingreso de la visita a la unidad de cuidados intensivos, de la información que recibían diariamente de la enfermera, el 100\% de los familiares la consideró clara y sencilla. Mencionaron que siempre eran informados previamente de cualquier exploración realizada o medio diagnóstico que requería su paciente, tanto dentro como fuera de la Unidad de Cuidados Intensivos.

Normalmente se concibe la información referente a aspectos médicos y no de Enfermería. En nuestra opinión las dos son complementarias y no excluyentes, por lo que se hace indispensable una adecuada coordinación de la misma por parte de todos los profesionales implicados en el cuidado de la salud del paciente. (Pérez, 2009).

Los resultados obtenidos muestran similitud con el estudio de Franco y Santana en relación con el tiempo de visita, el tiempo le parecía inadecuado al $17 \%$ de los familiares; el $35 \%$ de los familiares no lo consideraba suficiente, y les gustaría que se incrementara. Con respecto al número ilimitado de familiares que pueden entran en la UCI durante las visitas, el $77 \%$ de los familiares entrevistados estaba satisfecho. El 75\% de los familiares expresó que el familiar estaría más cómodo si se pudiera sentar para permanecer con su familiar durante la visita.

En relación a las visitas, consideraron que el horario es adecuado, que es suficiente y están satisfechos de que puedan visitar a su familiar un número de parientes ilimitado. De todas formas, existe un porcentaje de familiares que pedía más tiempo de permanencia con su ser querido, lo cual se puede considerar en determinados pacientes donde la carga de enfermería no sea excesiva y que el paciente se beneficie de esta estrategia.

En líneas generales son las enfermeras quienes modifican la pauta de visitas establecida, tanto flexibilizándola como restringiéndola, y en algunos 
trabajos se ha demostrado que muchas de ellas piensan que la visita abierta beneficia a los familiares. (Franco, 2004).

Los resultados del estudio ofrecen satisfacción, ya que se cumplen las expectativas y necesidades de los propios familiares, no obstante es seguro que existen oportunidades de mejora, ya que el incremento en la calidad y la cantidad de la comunicación enfermera(o) - paciente y la introducción de ciertos cambios, como flexibilizar el horario de visitas en algunos pacientes, puede conducir a una mayor satisfacción de los familiares de pacientes ingresados en la UCI. Posteriormente será posible utilizar estos datos de satisfacción en iniciativas de mejoría de la calidad de los cuidados críticos.

El trabajo de investigación reafirma la solidez del apoyo psicosocial que brinda la enfermera en la unidad de cuidados intensivos, no solo a nivel asistencial en relación al cuidado de sus pacientes, además de ello, a nivel emocional, instrumental y en la solución de problemas conjuntamente con los familiares que asisten diariamente a la visita en los hospitales u otras instituciones de salud.

\section{Declaración de financiamiento y de conflicto de intereses:}

El estudio fue financiado por la autora, quien declara no tener algún tipo de conflicto de interés en la investigación realizada.

\section{Correspondencia:}

Manuel Reyes Tiburcio

Hospital Alberto Sabogal de EsSalud - Av. Arenales 1402, Jesús María- Lima, Perú.

Correo electrónico: mreyes_89@hotmail.com

\section{REFERENCIAS BIBLIOGRÁFICAS}

Bacigalupo, J. C., Uzal, M. (2000, Abril). La familia del paciente internado en la Unidad de Cuidados Intensivos; Rev. Med. 136(4): 53. Uruguay.

Concha, D., Sánchez, P., Lastra, P. (2004). Relación entre enfermeras de Unidades de Cuidados Intensivos $y$ familiares: Indicios para el cambio. Nurse investigation. 3 (1). Recuperado de http:// www.fuden. es/FICHEROS ADMINISTRADOR/ORIGINAL/ Original3.pdf

Machado, E., Spiri, C. 2007. Dimensión personal de Trabajo del proceso para las enfermeras de la Unidad de Cuidados Intensivos. Brasil.

Martínez, P. 2005. Calidad de atención en la Unidad de Cuidados Intensivos; Hospital Nacional Edgardo Rebagliati Martins - ESSALUD. Lima - Perú.

Rodríguez, M. 2006. Implicación familiar en los cuidados básicos del paciente crítico. Unidad de Cuidados Intensivos del Hospital Universitario Insular de Gran Canaria. España.

Rosa, F. 2003. Percepción familiar del paciente crítico en la Unidad de Cuidados Intensivos; Hospital Nacional Edgardo Rebagliati Martins.
Ruiz, M. 2002. Satisfacción del familiar con el cuidado a pacientes en estado crítico. Universidad Autónoma de Nueva León, México.

Torres, L., Morales, J.M. 2004. Participación familiar en el cuidado del paciente crítico. Sociedad Andaluza de Enfermería de Cuidados Críticos. Andalucía - España.

Triviño, F., Torres, M. 2007. Satisfacción de expectativas de familiares con el cuidado de enfermería en una Unidad de Cuidados Intensivos de una clínica privada de Cali. Cuaderno de Investigaciones Fundación Universitaria del Área Andina No. 2 Vol. 2 - 76 p.

Uribe, E., Muñoz, M., Restrepo, J. (2004, Septiembre). Percepción familiar del paciente crítico cardiovascular. Investigación y Educación en Enfermería, vol. XXII, núm. 2, pp. 5061 Universidad de Antioquía Medellín, Colombia.

Velasco, J. 2006. Detección de necesidades de los familiares de pacientes ingresados en Unidades de Cuidados Intensivos; Unidad de Cuidados Intensivos Hospital Costa del Sol. Marbella, España.

Recibido: 10/04/2013

Aceptado: 30/09/2013 